\title{
BMJ Open Quality Improving population health in resident clinics: increasing pneumonia vaccination rates in adults 65 years and older
}

\section{Sheila McGreevy, Caylin McGowan, Kristin Gillenwater, Rebecca Opole, Meena Veluri, Kim Stehle, Bethany Ramm, Cheryl Gibson}

To cite: McGreevy S, McGowan C, Gillenwater K, et al. Improving population health in resident clinics: increasing pneumonia vaccination rates in adults 65 years and older. BMJ Open Quality 2020;9:e000830. doi:10.1136/ bmjoq-2019-000830

Received 16 September 2019

Revised 2 October 2020

Accepted 8 October 2020

\section{Check for updates}

(C) Author(s) (or their employer(s)) 2020. Re-use permitted under CC BY-NC. No commercial re-use. See rights and permissions. Published by BMJ.

Internal Medicine, University of Kansas Medical Center, Kansas City, Kansas, USA

Correspondence to Dr Sheila McGreevy; smcgreevy@kumc.edu

\section{ABSTRACT}

Streptococcus pneumoniae is a significant bacterial pathogen, especially in the elderly. There are two types of pneumococcal vaccines, one with polysaccharides from the capsule of 23 serotypes (PPSV23) and one with polysaccharides from 13 serotypes that have been conjugated to a protein (PCV13). Both vaccines decrease the incidence of invasive pneumococcal disease and are recommended for all people $\geq 65$ years of age. We found the vaccination rate against $S$. pneumoniae in patients $\geq 65$ years of age in our resident physician continuity clinics was $59.85 \%$, which is considerably lower than the vaccination rate of $69.16 \%$ in our attending physician clinics. The aim of our study was to double the number of vaccinations given in the resident physician clinics over a 1-month period without compromising workflow or unduly burdening the rooming nurse or resident physicians. For our primary intervention, we assigned a designated nurse with expertise in vaccinations the task of reviewing charts ahead of clinic visits then pending the order for the appropriate pneumococcal vaccine. Our secondary intervention was the education of physicians through pocket cards, verbal encouragement and email reminders. $\chi^{2}$ test was conducted to compare the proportion of patients 65 years or older who were vaccinated between the intervention and control groups. The results indicated that the intervention group patients were 2.61 times $(95 \% \mathrm{Cl} 1.18$ to 6.10$)$ more likely to be vaccinated compared with the control group patients ( $20 \%$ vs $8.7 \%$, $\chi^{2}(1)=5.16, p=0.02$, Cramer's $\left.V=0.16\right)$. The intervention resulted in more than doubling the number of vaccinations in the intervention group compared with the control group. Moreover, poststudy interviews and surveys indicated our workflow is sustainable and amendable to wider use within the resident physician clinics.

\section{PROBLEM}

An audit during the calendar year of 2018 of the General Internal Medicine ambulatory clinic revealed the pneumococcal vaccination rates for patients 65 and older were lower than target rates. Furthermore, there was a gap in the care between vaccination rates for the group of patients seen by experienced physicians on the medical staff at our institution ('attending physicians') and the physicians still in training ('resident physicians'). In the attending patient panels, $69.16 \%$ of the patients received at least one of the two available vaccines, and in the resident physician patient panels, $59.85 \%$ of the patients received the vaccine (table 1 ). In table 1 , the Initial Population represents patients seen in the Internal Medicine Clinic 65 years of age and older. 'Met' represents patients who have ever received either PPSV23 or PCV13, and 'Not Met' represents patients who have not received a pneumococcal vaccine. Excluded are patients whom the provider deemed as not needing to receive the pneumococcal vaccine.

After determining our resident physicians had low vaccination rates when compared with the attending physicians in 2018, we conducted a retrospective 7-day audit of residents in 2019. We found numerous missed opportunities for pneumonia vaccinations. In response, we proposed to address this critical gap through development and implementation of a mixed method quality improvement (QI) project, leveraging evidence-based strategies to enhance vaccination rates in the resident continuity clinics. The goal of our QI project was to improve the process for ordering and administering pneumonia vaccines in the resident physician continuity clinics, such that we would double the number of Streptococcal pneumoniae vaccines given to patients seen in resident physician clinics over a 1-month period. We aimed to devise a workflow that was sustainable and reproducible, so that it could be used long term as part of the clinic workflow and eventually close the gap between attending physician and resident physician vaccination rates.

\section{BACKGROUND}

$S$. pneumoniae continues to be an important cause of morbidity and mortality among the 


\begin{tabular}{|c|c|c|}
\hline $\begin{array}{l}2018 \text { performance for } \\
\text { either PVC13 or PPSV23 } \\
\text { documentation for patients } \geq 65 \\
\text { years of age }\end{array}$ & IM resident & IM attending \\
\hline Initial population & 926 & 3795 \\
\hline Met & 553 & 2612 \\
\hline Not Met & 371 & 1168 \\
\hline Excluded & 2 & 18 \\
\hline Number vaccinated (\%) & $59.85 \%$ & $69.16 \%$ \\
\hline
\end{tabular}

IM, Internal Medicine.

elderly. ${ }^{1}$ Vaccines are an important part of invasive pneumococcal disease prevention. The incidence of invasive pneumococcal disease is decreasing, likely due to the introduction of conjugated pneumococcal vaccinations in both paediatric and adult populations. Overall, invasive pneumococcal disease in the USA in adults 65 or older decreased from 59 cases per 100000 people in 1998 to 23 cases per 100000 people in $2015 .^{2}$

Accordingly, one of the objectives for improving the health of all residents of the USA, as defined in the federal government's Healthy People 2020 initiative, is to increase the percentage of non-institutionalised adults $>65$ years old who have received at least one vaccine against $S$. pneumoniae from a baseline rate of $60 \%$ in 2008 to $90 \%$ in $2020 .^{3}$ A systematic review and meta-analysis of studies designed to improve influenza and pneumococcal vaccination rates supports the use of non-physician ambulatory team members to spearhead vaccination efforts, which is consistent with the team-based model of ambulatory care embedded in medical home initiatives. ${ }^{4}$ This review and meta-analysis concluded QI interventions can improve vaccination rates, although modestly, among community dwelling adults by implementing interventions that designate responsibility for vaccinations to non-physician staff or by having clinic staff members hand out information to eligible patients about pneumococcal vaccinations before their appointments.

There have been other successful approaches to improving vaccination rates in clinic settings. For example, at Brigham and Women's Hospital in Boston, the Department of Medicine QI team implemented a pneumococcal vaccine initiative in pulmonary and rheumatology clinics that lasted several years, using three intervention strategies: physician reminders, patient letters and a nurse driven model. ${ }^{5}$ In addition to these strategies, both division and individual provider-level performance data were provided to frontline staff by email monthly to encourage further increases in vaccination rates. Quarterly presentations were also held to review performance data and to assess process improvement efforts within the practices. Through these efforts, the pneumococcal vaccine rates showed steady improvement from the beginning of the Intervention in February 2009 (52\% pulmonary; 50\% rheumatology) to January 2015 (79\% pulmonary; 87\% rheumatology).

Similarly, an integrated approach was implemented to improve immunisation rates in a busy ambulatory setting by using staff education with audit, feedback and incentives and increasing patient demand through messages delivered by staff and information flyers. ${ }^{6}$ This QI project was able to achieve a statistically significant difference between the preintervention pneumococcal immunisation rate of $24 \%$ and the postintervention rate of $47 \%$. Other successful approaches have involved creating best practice reminder alerts in the electronic medical record to prompt physicians to order vaccinations and inform them of contraindications, making it easier and more efficient compared with their previous processes, ${ }^{7-10}$ addressing patient fears and concerns about the safety or adverse effects of the vaccine ${ }^{11}$ and using telephone reminders to increase patient demand. ${ }^{12} 13$

Vaccination against $S$. pneumoniae is a quality metric tracked in the General Internal Medicine ambulatory clinics at our academic institution. Within General Internal Medicine clinics, there are clinics where experienced physicians who are part of the medical staff of the institution ('attending physicians') see their own panel of patients, and clinics where physicians in training ('resident physicians') see patients under the supervision of an attending physician. Our academic resident training programme uses the 3 plus 1 schedule. As such, for 1 week out of every 4 week cycle, resident physicians see patients in outpatient continuity clinics. Each resident physician has a panel of approximately 90 patients they follow over their 3-year residency. During their week of continuity clinic, the resident physicians see patients during four half day clinic sessions with approximately six patients per session, under the supervision of an attending physician.

In both attending physician clinics and resident continuity clinics, adult patients are seen for three primary visit types: acute problems, chronic disease management and preventive care. Immunisations can be addressed at any of these visit types.

Vaccines are generally ordered by the physician through the electronic medical record. A nurse administers the vaccine. Administration of the vaccine is documented in the electronic medical record (EMR) and via a paper consent form, which is signed by the patient and scanned into the chart. The two vaccines studied, PPSV23 and PCV13, are readily available in our clinic. The patients do not need to pay a fee at the time of the vaccination. If the patient is insured, insurance will generally pay the fee associated with the vaccination.

We focused our QI project on pneumonia vaccine administration in our ambulatory clinic; however, patients do have opportunity for vaccination outside the clinic setting. In the USA, vaccines are available in pharmacies and during hospitalisations for acute illness. However, many patients still get their vaccines during routine ambulatory office visits. Our electronic medical 

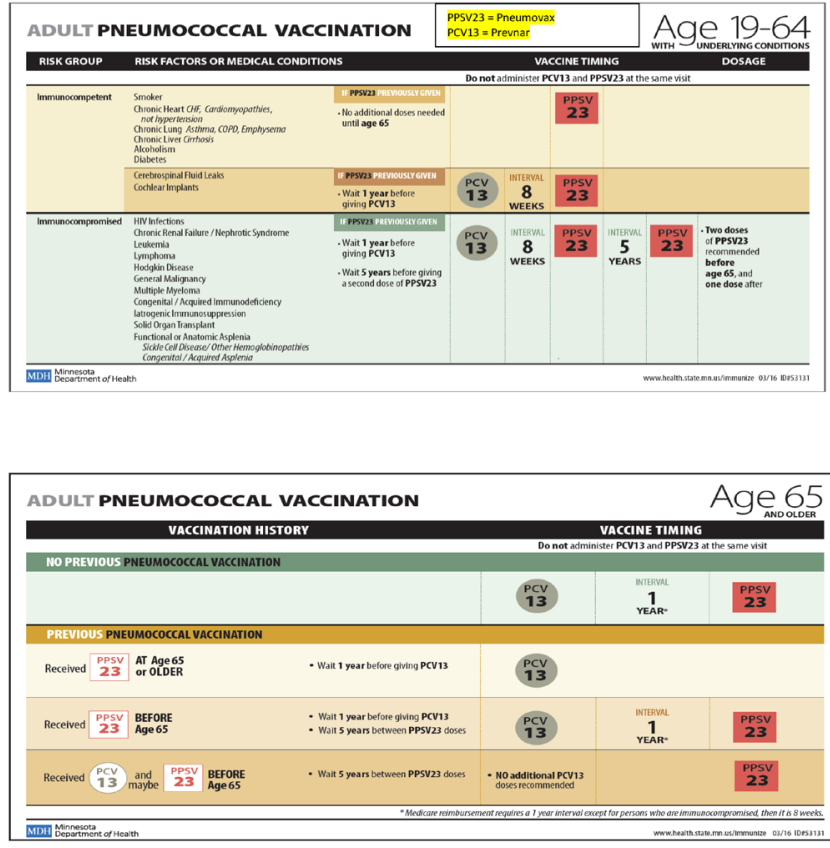

Figure 1 Pocket cards used in educational outreach 2018.

record captures many of the vaccinations performed in other settings.

We chose to focus this project on pneumonia vaccination as opposed to other vaccinations, because each adult vaccine has different barriers, including variability in cost, insurance reimbursement, vaccine availability, number of shots in the recommended schedule and target age range. We chose to limit our scope to one vaccine process and one set of barriers.

\section{MEASUREMENT}

We measured the number of conjugated (PCV13) and polysaccharide (PPSV23) pneumococcal vaccines given to patients seen in resident physician continuity clinics over 4 weeks. Each week, 12-16 residents see patients under the supervision of 3-4 attendings. We chose a group of eight residents (two groups of four) each of the 4 weeks for our intervention. We compared the number of vaccines ordered by these residents to the residents in the control group. Because of the complexity of tracking vaccines administered, we used two different data sources and cross checked our data across the two sources. Our primary data source was the paper vaccination consent form, signed by the patient at the time of the vaccination. We cross referenced the number of consent forms with our second data source, the EMR. We did a manual review of data in Epic, our EMR provider, looking at the order and vaccine administration history.

\section{DESIGN}

The precursor to the QI project described in this paper was a series of educational sessions done in 2017-2018 regarding the importance of the pneumococcal vaccines and the details of the recommended vaccine schedule, as defined by the United States Advisory Committee on Immunization Practices (ACIP). Pocket cards were distributed to all the nurses and physicians in the clinic (figure 1). ${ }^{14}$ In a series of meetings, we discussed the ACIP guidelines for pneumococcal immunisation with all the physicians and nurses. Pre-education and posteducation surveys were not done. We received positive verbal feedback regarding the pocket cards; however, we were unable to demonstrate improvement in the vaccination rates. We found the data difficult to track for reasons as varied as difficulty differentiating between the two pneumonia vaccines in the EMR, lack of baseline data, trouble identifying an appropriate denominator and challenges with our vaccine inventory and consent form documentation. Also, our educational outreach may not have been a robust enough intervention to result in significant benefit. Our current QI project is designed to build on the 2018 educational endeavour, incorporating remedies for the weaknesses of the initial effort.

Our current QI team includes stakeholders from multiple disciplines, including physician leaders, nurse champions, research mentors and front desk clinic staff. We mapped the current clinic workflow for vaccinations with all the stakeholders present and then created a fishbone analysis of barriers to vaccination. Our study design involved a mixed method approach to evaluate the impact of the intervention on vaccination rates and the nurses' and residents' experiences with implementation. Our intervention had several components. The primary intervention was pending the order for the vaccine prior to the office visit, such that the order was ready for the resident physician to sign during the office visit. Pending of the appropriate vaccination order was done by designated clinic nurses with expertise in vaccinations who reviewed patient charts prior to the visit. If the patient was $\geq 65$ years old and due for either PCV13 or PPSV23, the nurse would pend the order for the appropriate vaccine within the EMR and then communicate this information to the rooming nurse with a flagged paper consent form. The rooming nurse would briefly discuss the vaccine with the patient and resident at the start of the visit. During the week prior to clinic, an email explaining the intervention and encouraging vaccination was sent to the two attending physicians and eight resident physicians who were part of the study group. On the first day of clinic, face to face education was provided to the rooming nurses, resident and attending physicians, with explanation of the project and encouragement to vaccinate. Each physician was given a pocket card with the ACIP pneumonia vaccine recommendations.

The interventions were chosen to overcome barriers identified in our fishbone analysis, especially barriers related to time constraints and confusion regarding the vaccination schedule. The time constraint was addressed by having another member of the team review the chart outside of the limited time available during the office 
Table 2 Patients seen who received pneumonia vaccinations

\begin{tabular}{lll}
\hline Variable & Intervention & Control \\
\hline Patients scheduled, all ages & 420 & 396 \\
Patients seen, all ages (scheduled patients, not including no shows) & 350 & 345 \\
\hline Patients seen $\geq 65$ & 95 & 103 \\
Patients seen $\geq 65$ who received a pneumonia vaccine & $19(20.00 \%)$ & $9(8.74 \%)$ \\
Patients seen $\geq 65$ who received a pneumonia vaccine with a pended order & $12(63 \%$ of given vaccines) & 0 \\
Patients seen $\leq 65$ & 255 & 242 \\
\hline Patients seen $\leq 65$ who received a pneumonia vaccine & $9(3.35 \%)$ & $5(1.45 \%)$ \\
\hline
\end{tabular}

visit. The complexity of the vaccination schedule was addressed through education and a readily available and readable point of care reference tool.

\section{RESULTS}

Over the course of 4 weeks, 350 patients were seen in the Intervention group, with $95(27.14 \%)$ of those patients $\geq 65$ years of age (table 2 ). In the control group, 345 patients were seen, with $103(29.85 \%) \geq 65$ years of age. In the intervention group, $19(20.00 \%)$ patients $\geq 65$ were given one of the pneumonia vaccines compared with $9(8.74 \%)$ in the control group $\left(\chi^{2}(1)=5.16, p=0.02\right.$, Cramer's V=0.16). The results indicated that the intervention group was 2.61 times (95\% CI 1.18 to 6.10 ) more likely to vaccinate compared with the control group. A secondary benefit was an increase in the number of vaccinations given to patients less than 65 years old $(3.35 \%$ vs $1.45 \%)$. More than half of the vaccines $(63 \%)$ ordered by the study group resident physicians were pended by the nurse in advance of the visit.

\section{POSTSTUDY}

After the study was completed, semistructured interviews of the designated nurses pending the vaccine were conducted to explore their perspectives about the intervention. The interviews were performed by a researcher who has extensive experience in qualitative methods. She was not involved in the clinic and prior to the intervention had never met the nurses. The interviews included the following domains: how the pneumonia vaccine project might have influenced the amount of time spent preparing for the patient visit and answering questions from residents or attending physicians, (2) physician response to the intervention, (3) implementation of a standard vaccine protocol for resident clinic and (4) challenges and ideas to support pneumonia vaccinations. Interviews were recorded on a digital recorder and then transcribed verbatim. Thematic data analysis was conducted following the Braun and Clark's approach. ${ }^{15}$ Initially, research team members analysed the transcripts separately, identifying keywords and noting recurring ideas and explanations for each domain and captured the sentiments of the nurses.
The analysis of the interviews revealed that the nurses did not feel the pneumonia vaccine project took too much time preparing for the patient or answering questions from the residents or attending physicians. The nurses' views about the length of preparation time was depicted succinctly by the following passage.

"It was not a huge influence. It takes me about five min to go through a patient's chart to figure out if they are needing a pneumonia vaccine or not. So not much. Yeah, not much added time to what we originally do or what we normally do anyway.... but the pending of the order, the looking through the patient chart does not take a whole lot of time."

When asked what they have noticed about the resident physicians' response to the intervention, the nurses indicated it was mixed. Some residents were described as enthusiastic while others were ambivalent.

"Some of them seemed very kind of gung-ho about it. Others we would remind them every morning and either half of the time it wouldn't be address or part of the project would go missing."

Nurses were asked what they thought about a standard vaccine protocol for resident clinic where a designated nurse reviews charts ahead of the visit and pends the order for the appropriate vaccine. Nurses believed that the protocol would be good to implement within the resident clinic. The following extract from one nurse adequately describes the perception of the nurses about a standard vaccine protocol.

"Very beneficial to patients, a lot less that the residents have to focus on. When patients come in, they (residents) have so many problems that they are dealing with that healthcare maintenance gets lost. So, if there's a nurse that can go through the patient chart, see if there's a pneumonia vaccine or any vaccine that they are due for and go ahead pend the orders for the residents, that is one less thing they must think of. "

Suggestions to improve the way in which the pneumonia vaccine protocol was implemented were minimal. Nurses stated that the process needed to become embedded into 
the normal routine for clinic staff, residents and attending physicians to become accustomed to the new process.

"...I think more than anything it would have to be just a repetitive thing. Like hey, this is the new standard now."

Other suggestions included the need to have a chief vaccine nurse officer or placing a hard stop before residents can close their notes on the patient to remind them to address vaccinations.

In addition to the nurse interviews, we invited attending and resident physicians to complete a brief online survey about their experience with and views about the QI project. An email message was sent to all attending and resident physicians who were involved in the QI project $(n=32)$, inviting them to complete the survey. A link to the online survey was included in the body of the email message. Unique identifiers were not required for completion of the survey. The seven-item physician survey included questions about their level of awareness of the vaccine QI project. The physicians were also asked how helpful they believed the intervention was to increase the vaccine rate among the elderly and how burdensome the intervention was to their typical clinic schedule. Physicians were also asked how supportive they were of this type of intervention and future interventions that might pend orders for the other preventative care measures. Finally, they were asked to specify whether the pneumonia vaccine project increased their awareness of the need for vaccinations for patients of all ages and if there was any information that would have been helpful to know before the project was implemented.

Twenty-four of the 32 resident and attending physicians completed the survey ( $75 \%$ response rate). When asked how helpful the intervention was to increase the pneumonia vaccine rate among the elderly, the majority found it to be somewhat to very helpful. No one reported the intervention to be burdensome to the typical clinic schedule. Ninety-two per cent of respondents indicated they strongly favour having a nurse review charts and pend the order for the appropriate pneumonia vaccine for eligible patient 65 years and older. Moreover, they also favoured this same nursing model process for other preventative care measures. To understand how this QI project might have influenced the residents' and attending physicians' awareness about vaccinations, we asked if the QI project had increased their awareness of the need for vaccinations for patients of all ages. Approximately $60 \%$ of respondents agreed or strongly agreed that the QI project increased their awareness while about a third of respondents indicated a neutral response. Only $8 \%$ of respondents reported the QI project did not increase their awareness of the need for vaccinations among patients of all ages.

Postintervention, when the nurses were no longer pending the vaccine prior to the visit, we counted the number of vaccines given in resident clinic to see if the positive effects of the study were sustained. For some of the residents, this was 4 weeks after the intervention, for others it was 8 weeks after the intervention. We found no sustained benefit overall, a high degree of variability in the number of vaccines given and many missed opportunities for vaccination. The intervention group gave a total of six pneumonia vaccines to patients $\geq 65$ years of age over a 4 -week period, which was $5.7 \%$ of patients $\geq 65$ seen, with 19 missed opportunities. The control group also gave six pneumonia vaccines to patients $\geq 65$, which was $4.6 \%$ of patients seen $\geq 65$, with 26 missed opportunities. The decrease in vaccination rates poststudy suggests the nurse pending the order in advance of the visit is crucial to the success of the intervention.

\section{LESSONS AND LIMITATIONS}

The surprising complexity of communication of our relatively simple intervention is a limitation. The chain of communication involved designated vaccine nurses, front desk staff, rooming nurse, patient, resident physician, attending physician and check out staff. The means of communication included EMR, paper and verbal hand offs. Glitches in communication were the main reason for missed opportunities for vaccination. Our next Plan-DoStudy-Act (PDSA) cycle will focus on improving communication with the rooming nurse and resident physician. Another significant limitation is the difficulty with data collection. Because our two data sources each had limitations, we cross checked the paper consent forms against the EMR documentation, with a review of individual charts to reconcile differences. This is time consuming and hard to sustain. While this was an important step in demonstrating our change led to improvement, it is not required for the process long term. Other limitations include high variability among different groups of physicians and rooming nurses in their enthusiasm for the project and vaccination rates, high variability in baseline vaccination rates and potential cross-contamination via sharing information about the vaccine project from the study group to the control group because of physical proximity within the clinic.

The strength of the project is the sustainability of the intervention. The amount of time it takes for the designated nurses to review charts ahead of the visit and pend the order was not burdensome and can be incorporated into the clinic workflow easily. The residents and attending physicians did not find the intervention burdensome and would favour continuing to have nurses pend the order for the vaccine prior to the visits. Our intervention can potentially be used to increase the rates of other preventive quality metrics as well. Another strength is the spillover effect our intervention had on vaccination rates for patients less than 65 who need the vaccine. Raising awareness of the vaccine for one age group also increased the vaccination rates for a different age group. 


\section{CONCLUSION}

In the USA, adults are more likely to die of vaccinepreventable diseases than children, yet fewer adults are fully vaccinated. We aimed to close this care gap by bringing vaccinations to the forefront of the busy office visit, using the expertise of a nurse champion who pends the order for the vaccine prior to the visit. With our intervention, patients were 2.61 times (95\% CI 1.18 to 6.10 ) more likely to be vaccinated against pneumonia, when compared with the control group.

Our results suggest that our strategy of enlisting the help of non-physician members of the care team is sustainable and effective. We hope to build on this project, by rolling out a similar project on a broader scale and by applying this same framework to other preventive quality metrics.

Acknowledgements The authors would like to acknowledge the efforts of Kody Stockton, Kelly Grasmick, Jamie Jones and Mary Washington who helped to execute portions of the quality improvement program described here.

Contributors SM designed the study as principal investigator and executed the interventions. She also contributed to the collection and analysis of data and drafted the manuscript. CM collected and analysed data, developed tables and participated in drafting the manuscript. KG, RO and MV participated in drafting the manuscript. $\mathrm{KG}, \mathrm{KS}$ and $\mathrm{BR}$ contributed to the overall study design and implementation and review of manuscript. CG advised on the data collection process, conducted and analysed the interviews and surveys, facilitated the overall data analysis and assisted with drafting the manuscript. All authors have reviewed and approve of the final manuscript.

Funding The authors have not declared a specific grant for this research from any funding agency in the public, commercial or not-for-profit sectors.

Competing interests None declared.

Patient and public involvement Patients and/or the public were not involved in the design, or conduct, or reporting, or dissemination plans of this research.

Patient consent for publication Not required.

Provenance and peer review Not commissioned; externally peer reviewed.

Data availability statement All data relevant to the study are included in the article.

Open access This is an open access article distributed in accordance with the Creative Commons Attribution Non Commercial (CC BY-NC 4.0) license, which permits others to distribute, remix, adapt, build upon this work non-commercially, and license their derivative works on different terms, provided the original work is properly cited, appropriate credit is given, any changes made indicated, and the use is non-commercial. See: http://creativecommons.org/licenses/by-nc/4.0/.

\section{REFERENCES}

1 Oligbu G, Fry NK, Ladhani SN. The pneumococcus and its critical role in public health. Methods Mol Biol 2019;1968:205-13.

2 CDC. Pneumococcal disease, surveillance and reporting, 2017. Available: https://www.cdc.gov/pneumococcal/surveillance.html

3 ODPHP. Immunization and infectious disease. Available: https://www. healthypeople.gov/node/4670/data_details [Accessed 30 Jun 2019].

4 Lau D, Hu J, Majumdar SR, et al. Interventions to improve influenza and pneumococcal vaccination rates among community-dwelling adults: a systematic review and meta-analysis. Ann Fam Med 2012;10:538-46.

5 Pennant KN, Costa JJ, Fuhlbrigge AL, et al. Improving influenza and pneumococcal vaccination rates in ambulatory specialty practices. Open Forum Infect Dis 2015;2:ofv119.

6 Clark RC, Jackson J, Hodges D, et al. Improving pneumococcal immunization rates in an ambulatory setting. J Nurs Care Qual 2015;30:205-11.

7 Karr JR, Lu JJ, Smith RB, et al. Using computerized physician order entry to ensure appropriate vaccination of patients with inflammatory bowel disease. Ochsner J 2016;16:90-5.

8 Ledwich LJ, Harrington TM, Ayoub WT, et al. Improved influenza and pneumococcal vaccination in rheumatology patients taking immunosuppressants using an electronic health record best practice alert. Arthritis Rheum 2009;61:1505-10.

9 Warner EA, Seleznick MJ. Using medical record reminders to improve pneumococcal vaccination rates. Jt Comm J Qual Saf 2004;30:331-4.

10 Dexter PR, Perkins S, Overhage JM, et al. A computerized reminder system to increase the use of preventive care for hospitalized patients. N Engl J Med 2001;345:965-70.

11 ACP Internist. Qi project helps raise vaccination rates, 2018. Available: https://acpinternist.org/archives/2018/02/qi- project-helpsraise-vaccination-rates.htm

12 Winston CA, Mims AD, Leatherwood KA. Increasing pneumococcal vaccination in managed care through telephone outreach. Am J Manag Care 2007;13:581-8.

13 Jacobson Vann JC, Jacobson RM, Coyne-Beasley T, et al. Patient reminder and recall interventions to improve immunization rates. Cochrane Database Syst Rev 2018;1:Cd003941.

14 Minnesota Department of Health. Adult pneumococcal vaccination, 2016. Available: https://www.health.state.mn.us/diseases/ pneumococcal/pocket.pdf

15 Braun V, Clarke V. Using thematic analysis in psychology. Qual Res Psychol 2006;3:77-101. 